\title{
Design and Implementation of Smart Cart using Labview
}

\author{
K Nishanth Rao, P.Ramesh, C.Ashokkumar, Ananya Bannu
}

\begin{abstract}
In this present generation most of the people prefer to visit supermarkets and hypermarkets to buy the products of their needs right from the food products to the household usage items. In general these hypermarkets and supermarkets provide trolleys to their customers to have a hand free convenient shopping. Though the entire shopping goes well most of the customers get stressed out by seeing those long queues and hours of waiting near the bill counters. Even after coming up with some temporary solutions for this highly time consuming problem like increasing the man power in bill counters it didn't bring a big difference. The aim of the paper is to overcome this problem with a permanent solution. Here we introduce a new system called "SMART CART" where we can most probably eliminate the time taking process at the counters. This ultimately leads to the customer satisfaction from the starting of the shopping till the end point. And also adds benefits like reduction of man power requirement and high efficiency with low expenses. In this competitive world of technology this brings a drastic change benefiting both customers and also retail industries by the usage of automated devices. The idea is implemented using LabVIEW software and hardware MyRIO.
\end{abstract}

Keywords: SMART CART, LabVIEW, MyRIO.

\section{INTRODUCTION:}

In the today's shopping method, the mall provides a trolley in which we can carry or drop all the items that are required to purchase [1]. After selecting our goods, we go to the billing counter to pay the amount. This process is time consuming and hectic. This requires more man power and the shops have to employ people to get the billing done at the counter.So, In order to eliminate those problems, we designed and implemented an application called "smart cart". This is mainly used to reduce the time taken at billing section, and also increase efficiency.This is processed, when the mobile application senses the product using camera, it sends the signal to the MyRIO via Bluetooth module. Consequently, the products add to the billing system and reducing the time consumed in the billing queue.

\subsection{Existing System:}

The existing supermarkets are employed to deal with the subsequent issues:

* Manual billing.

* Human staff is needed for billing.

Revised Manuscript Received on December 30, 2019.

* Correspondence Author

K Nishanth Rao, Department of Electronics and Communication Engineering, MLR Institute of Technology, Hyderabad, India

P.Ramesh, Department of Electronics and Communication Engineering MLR Institute of Technology, Hyderabad, India

C.Ashokkumar, Department of Electronics and Communication Engineering MLR Institute of Technology, Hyderabad, India

Ananya Bannu, Department of Electronics and Communication Engineering MLR Institute of Technology, Hyderabad, India

(c) The Authors. Published by Blue Eyes Intelligence Engineering and Sciences Publication (BEIESP). This is an open access article under the CC BY-NC-ND license (http://creativecommons.org/licenses/by-nc-nd/4.0/)
* Long waiting time for checkout.

* Getting product information is difficult and time consuming.

\section{PROPOSED SYSTEM:}

The main aim of a proposed paper is to provide a high technology oriented [2-3], affordable, robust system for aiding shopping in customers. The proposed, mart shopping cart is designed to enrich the overall shopping experience for persons in the supermarkets and malls.

Here we primarily use hardware components in the paper such as Bluetooth (HC-05), MyRio [1], and PIR sensor. Role of MyRio is to build a inter-connection between hardware and software. Where PIR sensor is used to reduce the theft conditions. The entirepaper application is built over the 'Android studio'. Initially we need to scan the particular trolley which we are going to carry. Here each and every trolley is assigned with a unique QR code[3]. Immediately as the QR code is scanned we can move ahead with our shopping. Then we need to attain a interference between customer and shopkeeper by connection application to MyRio [4] (HC-05) via Bluetooth.

Once the Bluetooth is connected we can either add or delete items by scanning the barcodes present on the products of our requirement. Finally after the completion of shopping we have a option of "I am done". Then we get a complete bill indicating details such as items and their cost including their quantity along with total price. Once the entire process is completed we can carry on with our final payment.

\section{DESIGN AND IMPLEMENTATION:}

In the proposed, Smart Cart (Fig.1) shows the block diagramand it consists of Central server, MyRIO, Sensor, Bluetooth module, and power supply as the major blocks. All these blocks are connected as shown in fig 2.

3.1 Bluetooth: HC-05 Bluetooth Module [8] is an easy to use Bluetooth Serial Port Protocol module, designed for transparent wireless serial connection setup. Serial Port Bluetooth module is fully qualified Bluetooth V2.0+EDR $3 \mathrm{Mbps}$ Modulation with complete $2.4 \mathrm{GHz}$ radio transceiver and baseband. It uses CSR Blue core 04-External single chip Bluetooth system with CMOS Technology.

3.2 Power Supply:A Power Supply [5] is a component that supplies power to at least one electric load. Typically, it converts one type of electrical power to another, but it may also convert a different form of energy such as solar, mechanical or chemical into electrical energy. A power supply provides components with electric power.

3.3 PIR Sensor: A PIR sensor [6] is an electronic sensor that measures infrared light radiating from objects in its field of view. 
They are most often used in PIR-based motion detectors. PIR sensors are commonly used in security alarms and automatic lighting applications.

PIR sensor detects general movement, but do not give information on whom or what moved.

3.4 MyRIO: NI myRIO-1900 gives simple info (AI), simple yield (AO), advanced information and yield (DIO), sound, and power yield in a reduced installed gadget. The NI myRIO-1900 associates with a host PC over USB and remote $802.11 \mathrm{~b}, \mathrm{~g}, \mathrm{n}$.

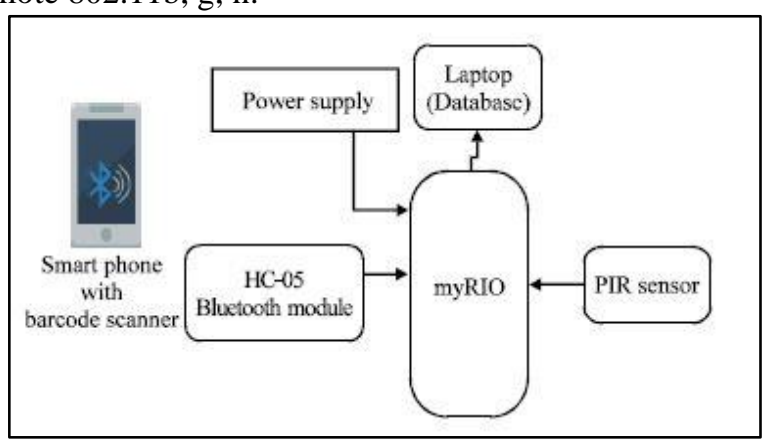

Fig 1: Block diagram of Smart Cart

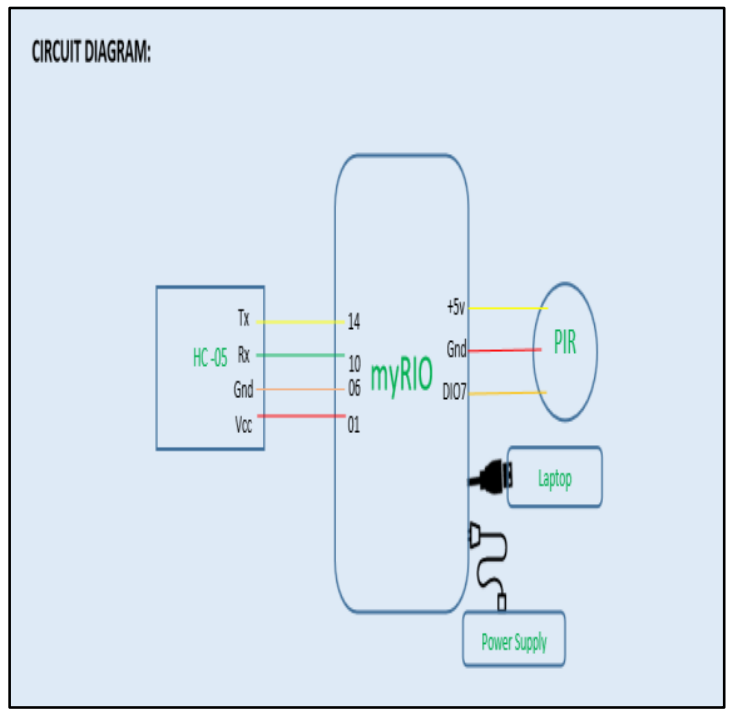

Fig 2: Circuit Diagram

\section{METHODOLOGY:}

The first part of the module is to store trolley ID, so the Bluetooth module is set to read state and when Bluetooth is connected to the application it receives a message "Trolley", with that shopping starts and whenever item is added using app, MyRIO receives ID's to store the items added to the cart by comparing ID's. Best part is security whenever the item is added; MyRio gives a chance to put a hand in it.

PIR detects the motion of the object where the access will be granted only when the barcode of the item is scanned either for adding item or deleting item and for all other cases access will be denied to add item or delete item. So, when the shopping is done, for verification, checkout phase starts where customer removes all the items from the basket by using PIR. Finally at the end of the shopping, Billing is automatically done and bill is stored in the database.

\subsection{Front Panel}

In the front panel, different types of controls and indicators are used like LED indicators as shown in fig 3.

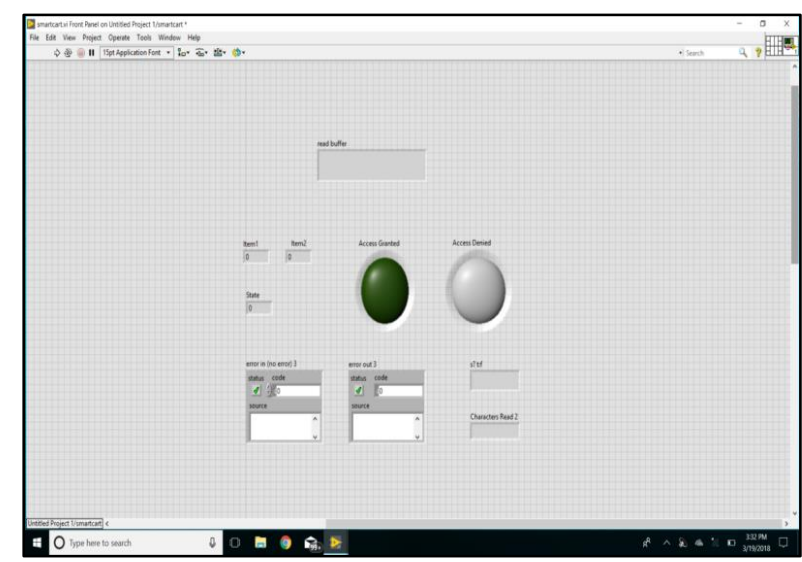

Fig 3: Front Panel in LabVIEW

When you open a new or existing VI, the front panel window of the VI appears. The front panel window is the user interface for the VI. fig3 shows an example of a front panel window.

The front panel shows the status of the PIR sensor whether the access is granted or denied by using the Boolean indicators and the increment of the items in the list when added and the decrement of the item from the list when deleted can be seen in the numerical indicators and Read buffer is used to see the trolley name which is scanned and error in and error out are used to indicate whether there is any error present in the code or not.

\section{RESULTS AND DISCUSSION}

Step1: Initially, We need to store the unique trolley [7-8] ID's and these ID's are assigned to their respective trolleys. There must be a inbuilt scanner or you need to download an application to support the barcode and QR code . Secondly, We need to download the SMART CART application in order to proceed further shopping as shows in fig 4.

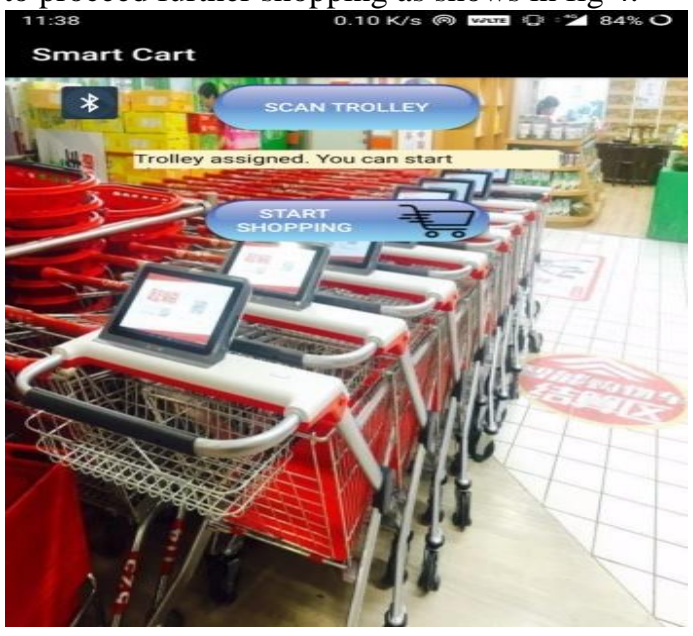

Fig 4: Assigning of Trolley

Step2: Next after trolley id are matched then it goes to next screen and there we need to connect the Bluetooth. The Bluetooth module we used is HC-05 Bluetooth module, it shows the fig 5 . 


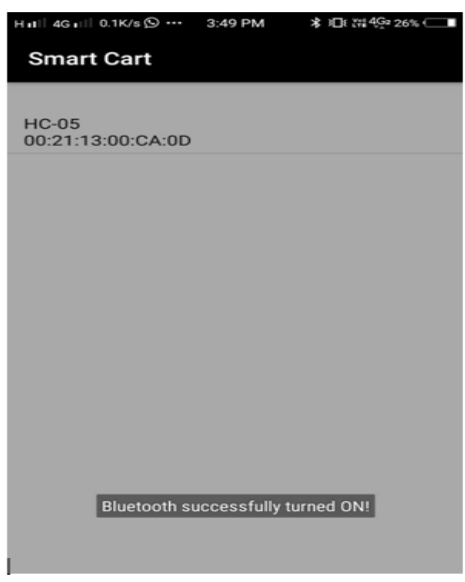

Fig 5: Connecting Bluetooth

Step3: After Bluetooth is connected, As shown in the fig 6 there will be two options i.e ADD or DELETE. When we press on the add or delete button, We need to scan the product barcode to add or delete the item from the list. Whenever product is added or deleted using app, myRIO receives IDs to store the added or deleted products to the cart by comparing IDs.

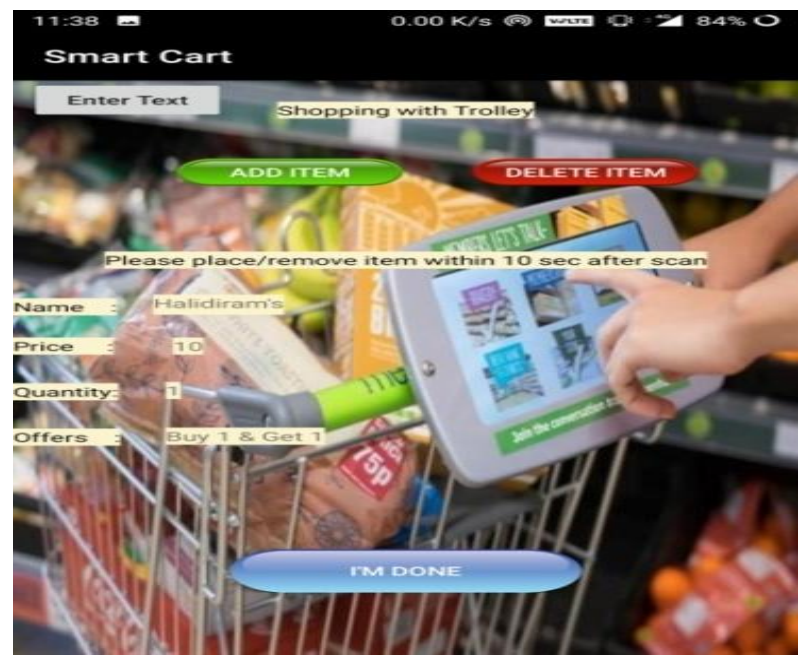

Fig 6: Displaying of the product added / deleted

Step4: The fig 7 Showing the bill and total items in the cart.

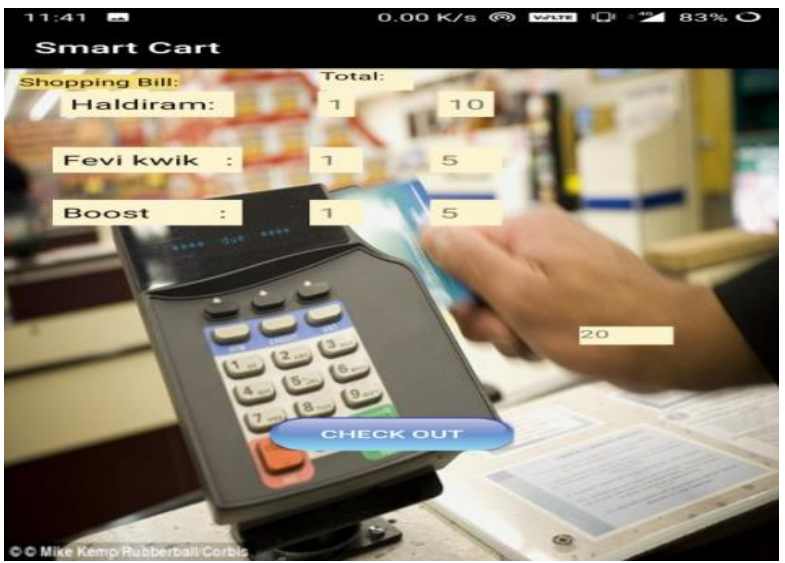

Fig 7: Bill Items in cart

Step5: The fig 8 shows that the, Click End Shopping

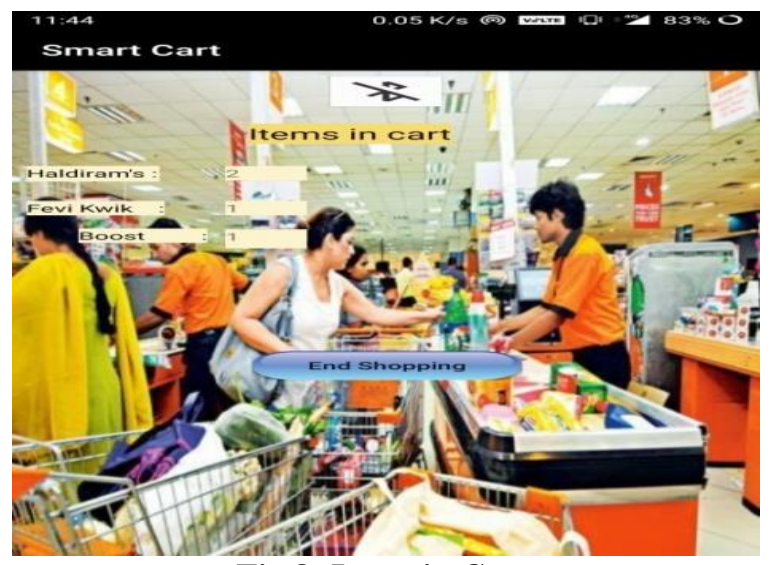

Fig 8: Items in Cart

\section{CONCLUSION}

The smart shopping cart is effortless to use, affordable price and do not need any special tutorials. The proposed system is obviously required for the real time marketing organizations to set up within the advancement technology, save time and man power. It generatesacomputerized central billing system for supermarkets. With the help of smart cart application customers will make the bill payment at the counters. Consequently, their products information is directly fetched to computerized central billing system.

\section{REFERENCES}

1. G. ShravanKumar,K. NishanthRao, "Monitoring of Relative Humidity in Soil Using LabVIEW", Journal of Advanced Research in Dynamical and Control Systems, 08-Special Issue-2018,pp.16401644 .

2. K. Haribabu, S.V.S. Prasad and M. Satish Kumar, 2018. An IOT Based Smart Home Automation Using LabVIEW. Journal of Engineering and Applied Sciences, Vol 13, Issue: 6, 2018, PP. : 14211424

3. Ch. Umasankar, Md. Abdul Rawoof, "Intelligent Agribot for Seed Sowing and Fertilizer Distribution Using Labview", Journal of Advanced Research in Dynamical and Control Systems, 08-Special Issue, 2018, PP.1657-1660.

4. Akshay Kumar, Abhinav Gupta, S.Balamurugan, S. Balaji, R.Marimuthu, "International conference on Microelectronic Devices, Circuits and Systems (ICMDCS)", 12 Aug. 2017.

5. Suryaprasad J, Praveen Kumar B O, Roopa D \&Arjun A K, “ A Novel Low-Cost Intelligent Shopping Cart," 2011 IEEE 2nd International Conference on Networked Embedded Systems for Enterprise Applications.

6. P.T. Sivagurunathan ,P. Seema, M. Shalini, R. Sindhu,“ SMART SHOPPING TROLLEY USING RFID,” International Journal of Pure and Applied Mathematics, Volume 118 No. 20 2018, 3783-3786

7. K.Gogila Devi, T.A.Kaarthik, N.KalaiSelvi, K.Nandhini, S.Priya, "Smart ShoppingTrolley Using RFID Based on IoT," International Journal of Innovative Research inComputer and Communication Engineering. Vol. 5, Issue 3, 2017.pp.5392-5398.

8. Chandrasekar.P , Ms. T. Sangeetha, " Smart Shopping Cart with Automatic Central Billing System through RFID and ZigBee," International Conference on Information Communication and Embedded Systems (ICICES2014).

\section{AUTHOR PROFILE:}

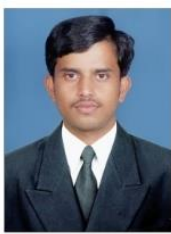

K Nishanth rao, Completed my B.Tech in the year of 2010 in Electronics and Communication Engineering, completed my M.Tech in the year of 2012 in RF \& Microwave Engineering, Presently pursuing my Doctoral Degree in Antennas in DSU, Bangalore, India. My Interested Domains are Electromagnetic fields, Antennas and Communications.

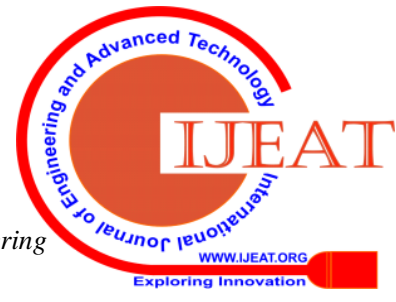


P Ramesh, Completed my B.Tech in the year of 2008 in Electronics and Communication Engineering, completed my M.Tech in the year of 2010 in VLSI. My Interested Domains are Electromagnetic fields, Antennas and Communications. 